\title{
ESSENTIAL OIL COMPOSITION AND ANTIMICROBIAL SCREENING OF SOME IRANIAN HERBAL PLANTS ON Pectobacterium carotovorum
}

\author{
MEHRSOROSH H. ${ }^{1}$ \\ GAVANJI S. ${ }^{2 *}$ \\ LARKI B. $^{3}$ \\ MOHAMMADI M.D. ${ }^{3}$ \\ KARBASIUN A. ${ }^{4}$ \\ BAKHTARI A. ${ }^{5}$ \\ HASHEMZADEH F. ${ }^{5}$ \\ MOJIRI A. ${ }^{6}$
}

\author{
${ }^{1}$ Technology Incubator Centre \\ Islamic Azad University, Khorasgan, Iran \\ ${ }^{2}$ Young Researchers and Elite Club \\ Khorasgan branch, Islamic Azad University, Khorasgan, Iran \\ ${ }^{3}$ Department of Plant protection \\ Khorasgan (Isfahan) branch, Islamic Azad University, Isfahan, Iran \\ ${ }^{4}$ Department of Agronomy and Plant Breeding \\ Khorasgan branch, Islamic Azad University, Khorasgan, Iran \\ ${ }^{5}$ Isfahan University Of Technology \\ Department of Animal Science, Iran, Animal Science \\ ${ }^{6}$ School of Civil Engineering, Engineering Campus \\ Universiti Sains Malaysia \\ 14300 NibongTebal, Penang, Malaysia
}

Received: 11/09/2013

Accepted: 19/01/2014

Available online: 21/01/2014

\begin{abstract}
In recent years, there has been growing concern regarding undesirable side effects of synthetic antimicrobial drugs used for food preservation or in medicine and an explosive global spreading of multidrug resistant microbes are considered as a substantial global health threat. This necessitates the searching for new classes of safe and more effective antimicrobial agent by acting with different mechanisms. This study aimed to determine the antimicrobial activity of essential oils of some herbs which are endemic Iranian plants using minimum inhibitory (MIC) concentration of their essential oils. Results obtained from minimum inhibitory concentration showed that the essential oil of Thymus vulgaris, compared to other extracts, possess the best inhibitory effect in the lowest concentration. The extracts of Artemisia kermanensis, Lavandula officinalis, Rosemarinous officinalis and Eucalyptus caesia are reported to have inhibitory effect on Pectobacterium carotovorum.
\end{abstract}

Keywords: Pectobacterium carotovorum, essential oil, minimum inhibitory concentration.

\section{Introduction}

In recent years, there has been growing concern regarding undesirable side effects of synthetic antimicrobial drugs/chemicals used for food preservation or in medicine and an explosive global spreading of multidrug resistant microbes are considered as a substantial global health threat (Telci et al., 2006). This necessitates the searching for new classes of safe and more effective antimicrobial agent by acting with different mechanisms. A number of plants containing secondary compounds could possess some of these ideal preservative characteristics mainly due to their antioxidant, antimicrobial and other biological potentials (Bakkali et al., 2008). In this regard, an increasing body of research was conducted on many herbal and culinary species in order to seek new natural bioactive compounds with 
special aims. Many pharmaceutical characteristics of aromatic plants are partially attributed to essential oils. Essential oils used in this study, including Thymus vulgaris, Artemisia kermanensis, Eucalyptus caesia Benth, Lavandula officinalis and Rosemarinous officinalis have been evaluated for their antimicrobial activities. Al-Bayati (Al-Bayati, 2008) claimed that thyme oil and methanolic extract had promising antibacterial activities against most pathogens. Kazemi and his coworkers investigated antimicrobial and antioxidant activities of the essential oil of Artemisia kermanensis against Bacillus cereus, Bacillus subtilis, Enterobacter spp, Klebsiellapneu moniaeand Escherichia coli. They reported that Artemisia kermanensis oil has maximum inhibition effect against Klebsiella pneumonia. In addition to these herbs, other investigated plants showed the same antimicrobial activity (Al-Bayati, 2008, Delaquis et al., 2002, Ghalem and Mohamed, 2008, Kazemi et al., 2011, Orhan et al., 2012). In many cases, it is reported that the yield and composition of the essential oil for each species have been affected by different factors such as physiological variations, environmental conditions, geographic variations and genetic factors (Di Pasqua et al., 2007, Takahashi et al., 2004). Pectobacterium carotovorum is a bacterium of the family Enterobacteriaceae; it formerly was a member of the genus Erwinia. The species is a plant pathogen with a diverse host ranges including potato, African violet, and other agriculturally and scientifically important plant species. It causes soft rot and blackleg of potato and vegetables, as well as slime flux on many different tree species. Echis is more frequent in subtropical and tropical climates and has a host range that includes carnation, leopoldlily, maize, pineapple, potato and African violet (Saintpaulia ionantha).The soft rot erwinias are found on plant surfaces and in soil where they may enter the plant via wound sites or through natural openings on the plant surface, e.g. lenticels. As this bacterium causes diseases in lots of different plants, so it is important to find safe and effective antibacterial agent for fighting with this bacterium. In these research different concentrations of Artemisia kermanensis Podl, Eucalyptus caesia, thymus vulgaris, Lavandula officinalis and Rosemarinous officinalis oils have been used against Pectobacterium carotovorum and the aim is to find the best oil and concentration that inhibit bacteria growth.

\section{Methodology}

\section{Plant materials and isolation of essential oils}

In this study we used fresh aerial parts of the herbs T. vulgaris, A. kermanensis, E. caesia Benth, L. officinalis and $R$. officinalis which were collected from Lorestan and Chaharmahal provinces (Iran) in 2012. The herbs were then dried at room temperature $\left(25^{\circ} \mathrm{C}\right)$ for 3 days. The dried herb samples $(500 \mathrm{~g})$ were ground and subjected to hydro distillation using a Clevenger-type apparatus. The oils were dried over anhydrous $\mathrm{Na}_{2} \mathrm{SO}_{4}$ and stored at $4{ }^{\circ} \mathrm{C}$ in a sealed amber vials until use (These vials can be used for one month).

\section{Oil analysis procedure}

Analysis was performed using GC-mass chromatograph with a HP-5MS column $(30 \mathrm{~m} \times 0.25 \mathrm{~mm}$, film thickness $0.25 \mathrm{~m}$ ). The carrier gas was helium at flow rate of $0.8 \mathrm{ml} \mathrm{min}^{-1}$. The column temperature was kept at $50{ }^{\circ} \mathrm{C}$ for 2 minutes and it was programmed to $200{ }^{\circ} \mathrm{C}$ at a rate of $3^{\circ} \mathrm{C} \mathrm{min}-1$ and kept constant at $200{ }^{\circ} \mathrm{C}$ for 10 minutes. Helium gas was used as carrier gas. The injection was performed in split mode with ratio of $50: 1$ at $250^{\circ} \mathrm{C}$. The compounds were identified by comparison of RRI (relative retention indices) with those reported in the literature and also by comparison of their mass spectra with published mass spectra (Adams, 2005; Sparkman, 1997). The retention indices for all the components were determined according to the Van Den Dool method using n-alkanes as standards (Van Den Dool and Kratz, 1963).

\section{Preparing bacterial strain and Dextrose Agar}

The bacterial strain was provided from Iranian Research Organization of Science and Technology. In this experiment Potato Dextrose Broth (PDB) and Potato Dextrose Agar (PDA) were taken from Merck Company. 


\section{Determination of inhibition of zone (IZ)}

In order to study the antimicrobial effect, Disk diffusion method was used. After $18 \mathrm{~h}$ of culture, liquid containing bacteria with standard density $\left(1 \times 10^{6} \mathrm{CFU} \mathrm{ml^{-1 }}\right)$ of $0.5 \mathrm{Mac}$ Farland in Potato Dextrose Broth (PDB) was prepared and $500 \mu \mathrm{l}$ of the liquid was transferred to Potato Dextrose Agar (PDA). The liquid was gently distributed on the surface of PDA using sterile loop. There have been blank disks with $6 \mathrm{~mm}$ in diameter containing $30 \mu \mathrm{l}$ with concentrations 25000, 50000, 300000, 100000 and $400000 \mathrm{ppm}$ on PDA. Disk containing antibiotics Gentamicin and Chloramphenicol was used as positive control and also disk containing $30 \mu \mathrm{l}$ of DMSO was used as negative control. The diameter of inhibition of zone was measured using caliper after $24 \mathrm{~h}$ of incubation at $37^{\circ} \mathrm{C}$ at the times 24.48 and $72 \mathrm{~h}$ in triplicate.

\section{Determination of MIC using dilution of wells}

Firstly in order to determine the minimum inhibitory concentration (MIC) of Pectobacterium carotovorum, the suspension of bacterial strain was prepared from liquid culture with standard darkness of $0.5 \mathrm{Mac}$ Farland. The essential oil which is diluted $10 \%$ with ethanol with primary concentration of $500 \mu \mathrm{g} \mathrm{ml}^{-1}$ was prepared and different dilutions (6 dilutions) were added to the pipes containing $10 \mathrm{ml}$ of liquid culture medium. MIC of essential oils was performed using Microwell method against bacterial strain (Sahin et al., 2004).Then the 96-well plate was used for determination of MIC. $95 \mu$ of Potato Dextrose Broth (PDB) and $5 \mu$ l of microbial suspension was added to every well. $100 \mu$ l of the essential oil with concentration of $500 \mu \mathrm{g} \mathrm{ml}^{-1}$ was added to the first well. Then $100 \mu \mathrm{l}$ was taken from the first well and it was transferred to the next well. This process went on to the 6th well. The last well was contained $195 \mu \mathrm{l}$ of PDB culture medium and also $5 \mu \mathrm{l}$ of microbial suspension without any essential oil. This well was considered as negative control. In the next step, the ingredients of every well were mixed using Rotary Shaker for $20 \mathrm{~min}$. Then it was put in an incubator for $24 \mathrm{~h}$ in an appropriate temperature $\left(37^{\circ} \mathrm{C}\right)$. The microbial growth was measured by spectrophotometer at $600 \mathrm{~nm}$ (Gavanji et al., 2011). In this study the effect of each essential oil was determined on Pectobacterium carotovorum separately with 3 replicates.

\section{Statistical analysis}

Before any statistical analysis, the normality of data and homogeneity of variances were evaluated. A factorial experimental technique has been used to investigate the types of plants, concentration of essential oil, interaction between types of plants and also concentration of essential oil. At second analysis, higher concentration of each plant was compared to two important antibiotics, CHEL and GEN through one way ANOVA (Minitab 16). Means of treatments were compared by Tukey's multiple comparison test. Differences were considered as significant at $\mathrm{P}<0.05$.

\section{Results and Discussion}

According to the results given in Table1, at three times of incubation, thymus vulgaris showed antimicrobial activity in a dose dependent manner and the most antimicrobial activity was observed at $300000 p p m$ ( $21.74 \mathrm{~mm}$ inhibition of zone). There was no significant difference at different times of incubation ( $p>0.10)$. A. kermanensis is another plant which was not effective at low doses (6.56 and 8.94 $\mathrm{mm}$ for 25000 and $50000 \mathrm{ppm}$ respectively), but moderate dose of the oil (10000 ppm) resulted in a considerable antimicrobial activity $(16.24 \mathrm{~mm})$ and the highest dose of inclusion showed the most effective antimicrobial property. The same antimicrobial property was observed for E. caesia, but the concentration $300000 \mathrm{ppm}$ was the most effective and increasing the concentration to $400000 \mathrm{ppm}$ led to exert lower antimicrobial activity $(19.20$ versus $18.03 ; \mathrm{p}=0.06)$. At the concentration $50000 \mathrm{ppm}$, two essential oils $L$. officinalis and $R$. officinalis showed a mild antimicrobial activity and increasing dose of the oil had improved this response and there was no difference between two higher doses $(p>0,10)$. 
Results obtained from MIC showed that the essential oils of $T$. vulgaris is equal to $145 \mu \mathrm{g} \mathrm{ml}^{-1}$ which has the best inhibitory effect with lowest concentration compared to other extracts. The extracts of $A$. kermanensis, L. officinalis, R.officinalis and E. caesia are reported to have inhibitory effect on Pectobacterium carotovorum respectively (Figure1).

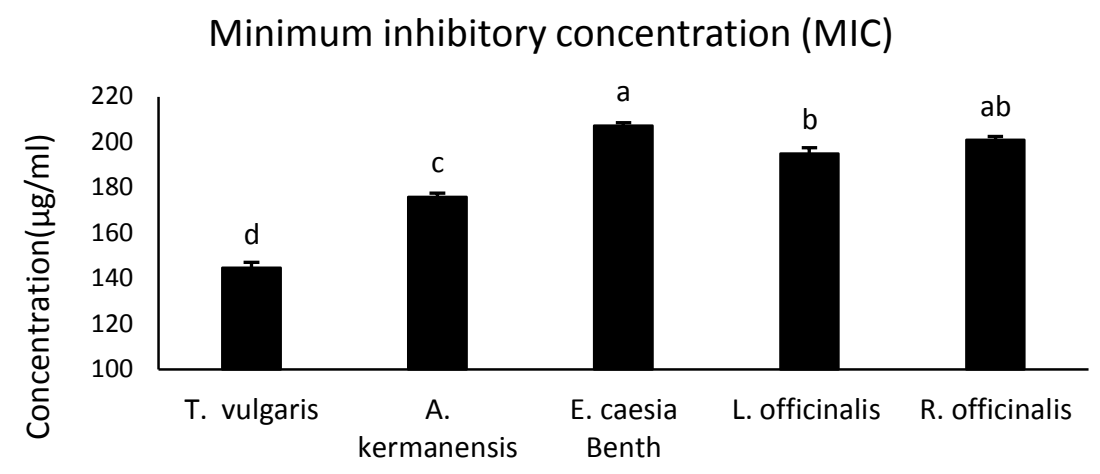

Plant's essence

Figure 1: Minimum inhibitory concentration (MIC) of extracts of herbal plants. Means with different letters show significantly different between treatment groups $(p<0.0001)$.

Table1. Antibacterial activity at different concentrations of some Iranian herbal plants.

\begin{tabular}{|c|c|c|c|c|}
\hline \multirow[b]{2}{*}{ Plant's essence } & \multirow[b]{2}{*}{ Con (ppm) } & \multicolumn{3}{|c|}{ Time(h) } \\
\hline & & 24 & 48 & 72 \\
\hline \multirow{5}{*}{ Thymus vulgaris } & 25000 & $7.05^{\text {ghi }}$ & $7.09^{\text {ghi }}$ & $7.14^{\text {ghi }}$ \\
\hline & 50000 & $13.71^{f}$ & $13.92^{\mathrm{e}}$ & $13.92^{\mathrm{de}}$ \\
\hline & 100000 & $17.96^{\mathrm{cd}}$ & $18.16^{\mathrm{bc}}$ & $18.36^{\mathrm{bc}}$ \\
\hline & 300000 & $21.47^{\mathrm{ab}}$ & $22.07^{\mathrm{a}}$ & $22.11^{\mathrm{a}}$ \\
\hline & 400000 & $22.41^{a}$ & $22.47^{\mathrm{a}}$ & $22.48^{a}$ \\
\hline \multirow{5}{*}{ Artemisia kermanensis } & 25000 & $6.56^{\mathrm{hi}}$ & $6.60^{\mathrm{hi}}$ & $6.73^{\text {ghi }}$ \\
\hline & 50000 & $8.94^{\mathrm{gh}}$ & $8.94^{\mathrm{fgh}}$ & $8.94^{\mathrm{fgh}}$ \\
\hline & 100000 & $16.24^{\mathrm{de}}$ & $16.43^{\text {cd }}$ & $16.41^{\mathrm{cd}}$ \\
\hline & 300000 & $16.86^{\mathrm{cd}}$ & $16.90^{\mathrm{C}}$ & $16.89^{c}$ \\
\hline & 400000 & $18.72^{c}$ & $18.84^{\mathrm{bc}}$ & $18.84^{\mathrm{bc}}$ \\
\hline \multirow{5}{*}{ Eucalyptus caesia Benth } & 25000 & $6.40^{i}$ & $6.40^{i}$ & $6.40^{i}$ \\
\hline & 50000 & $6.61^{\text {hi }}$ & $6.61^{\mathrm{hi}}$ & $6.61^{\text {hi }}$ \\
\hline & 100000 & $13.57^{f}$ & $13.58^{\mathrm{e}}$ & $13.80^{\mathrm{e}}$ \\
\hline & 300000 & $19.20^{\mathrm{bc}}$ & $19.42^{\mathrm{b}}$ & $19.45^{b}$ \\
\hline & 400000 & $18.03^{\mathrm{cd}}$ & $18.22^{\mathrm{bc}}$ & $18.23^{\mathrm{bc}}$ \\
\hline \multirow{5}{*}{ Lavandula officinalis } & 25000 & $6.42^{i}$ & $6.43^{i}$ & $6.43^{\mathrm{hi}}$ \\
\hline & 50000 & $9.16^{g}$ & $9.18^{\mathrm{fg}}$ & $9.22^{\mathrm{fg}}$ \\
\hline & 100000 & $12.34^{f}$ & $12.38^{\mathrm{e}}$ & $12.40^{\mathrm{e}}$ \\
\hline & 300000 & $17.84^{\mathrm{cd}}$ & $17.97^{\mathrm{bc}}$ & $17.98^{\mathrm{bc}}$ \\
\hline & 400000 & $18.35^{\mathrm{cd}}$ & $18.49^{\mathrm{bc}}$ & $18.50^{\mathrm{bc}}$ \\
\hline \multirow{5}{*}{ Rosemarinous officinalis } & 25000 & $6.67^{\text {hi }}$ & $6.67^{\text {hi }}$ & $6.67^{\text {hi }}$ \\
\hline & 50000 & $9.42^{\mathrm{g}}$ & $9.70^{f}$ & $9.75^{f}$ \\
\hline & 100000 & $13.87^{\mathrm{ef}}$ & $14.22^{\mathrm{de}}$ & $14.32^{\mathrm{de}}$ \\
\hline & 300000 & $17.85^{\mathrm{cd}}$ & $17.92^{\mathrm{bc}}$ & $17.97^{\text {bc }}$ \\
\hline & 400000 & $18.10^{\mathrm{cd}}$ & $18.18^{\mathrm{bc}}$ & $18.28^{\mathrm{bc}}$ \\
\hline SEM & & 0.44 & 0.45 & 0.46 \\
\hline$P$ values & & $<0.0001$ & $<0.0001$ & $<0.0001$ \\
\hline R-Square & & 0.99 & 0.98 & 0.98 \\
\hline CV (\%) & & 5.54 & 5.67 & 5.76 \\
\hline
\end{tabular}

Means with the same letters in each column are not significantly different at $p<0.05$.

Con: Concentration of plant's essence 
Table2. Compositions of Thymus vulgari

\begin{tabular}{|c|c|c|c|c|c|c|c|c|}
\hline No & Compositions & $\%$ & $\mathbf{R I}$ & $\mathrm{Sn}+1$ & Sn & RT & $\mathrm{Cn}+1$ & $\mathrm{Cn}$ \\
\hline 1 & alpha-thujene & 0.05 & 930 & 5.349 & 3.39 & 3.97 & 10 & 9 \\
\hline 2 & ALPHA-PINENE & 3.19 & 937 & 5.349 & 3.39 & 4.11 & 10 & 9 \\
\hline 3 & Verbenene & 0.03 & 956 & 5.349 & 3.39 & 4.488 & 10 & 9 \\
\hline 4 & Sabinene & 0.51 & 975 & 5.349 & 3.39 & 4.86 & 10 & 9 \\
\hline 5 & BETA-PINENE & 0.12 & 979 & 5.349 & 3.39 & 4.94 & 10 & 9 \\
\hline 6 & Heptanol & 0.08 & 988 & 5.349 & 3.39 & 5.117 & 10 & 9 \\
\hline 7 & BETA-MYRCENE & 0.18 & 992 & 5.349 & 3.39 & 5.197 & 10 & 9 \\
\hline 8 & gama-terpinene & 0.33 & 1006 & 7.991 & 5.349 & 5.518 & 11 & 10 \\
\hline 9 & alpha-terpinen & 0.07 & 1017 & 7.991 & 5.349 & 5.81 & 11 & 10 \\
\hline 10 & p-Cymene & 0.21 & 1025 & 7.991 & 5.349 & 6.004 & 11 & 10 \\
\hline 11 & LINALOOL & 20.2 & 1032 & 7.991 & 5.349 & 6.187 & 11 & 10 \\
\hline 12 & Ocimene & 0.7 & 1036 & 7.991 & 5.349 & 6.313 & 11 & 10 \\
\hline 13 & Dimethylstyrene & 0.12 & 1087 & 7.991 & 5.349 & 7.658 & 11 & 10 \\
\hline 14 & LINALOOL & 0.57 & 1099 & 7.991 & 5.349 & 7.961 & 11 & 10 \\
\hline 15 & BETA.-THUJONE & 0.06 & 1106 & 11.075 & 7.991 & 8.161 & 12 & 11 \\
\hline 16 & alpha.-Campholen & 0.21 & 1124 & 11.075 & 7.991 & 8.734 & 12 & 11 \\
\hline 17 & Mentha-2-en-1-ol & 0.59 & 1132 & 11.075 & 7.991 & 8.985 & 12 & 11 \\
\hline 18 & Trans Limonene Oxide & 0.39 & 1135 & 11.075 & 7.991 & 9.071 & 12 & 11 \\
\hline 19 & Camphor & 0.37 & 1142 & 11.075 & 7.991 & 9.283 & 12 & 11 \\
\hline 20 & Menthol & 0.13 & 1170 & 11.075 & 7.991 & 10.15 & 12 & 11 \\
\hline 21 & Terpene-4-ol & 0.5 & 1174 & 11.075 & 7.991 & 10.26 & 12 & 11 \\
\hline 22 & Naphtalene & 0.12 & 1177 & 11.075 & 7.991 & 10.38 & 12 & 11 \\
\hline 23 & alpha.-Terpineol & 0.66 & 1191 & 11.075 & 7.991 & 10.80 & 12 & 11 \\
\hline 24 & Carvacrol & 43.4 & 1209 & 14.282 & 11.075 & 11.37 & 13 & 12 \\
\hline 25 & trans-Carveol & 1.73 & 1216 & 14.282 & 11.075 & 11.59 & 13 & 12 \\
\hline 26 & Neral & 1 & 1238 & 14.282 & 11.075 & 12.28 & 13 & 12 \\
\hline 27 & Carvone & 1.45 & 1240 & 14.282 & 11.075 & 12.35 & 13 & 12 \\
\hline 28 & Geraniol & 0.4 & 1250 & 14.282 & 11.075 & 12.67 & 13 & 12 \\
\hline 29 & 2.6-Octadiena & 1.51 & 1266 & 14.282 & 11.075 & 13.19 & 13 & 12 \\
\hline 30 & METHYLBENZOATE & 0.18 & 1268 & 14.282 & 11.075 & 13.27 & 13 & 12 \\
\hline 31 & Isomenthone & 4.92 & 1286 & 14.282 & 11.075 & 13.83 & 13 & 12 \\
\hline 32 & LimonelAlchol & 1.9 & 1292 & 14.282 & 11.075 & 14.02 & 13 & 12 \\
\hline 33 & gamma. 1-cadinene & 0.08 & 1343 & 17.378 & 14.282 & 15.61 & 14 & 13 \\
\hline 34 & Eugenol & 0.08 & 1351 & 17.378 & 14.282 & 15.85 & 14 & 13 \\
\hline 35 & .alpha.-Copaene & 0.32 & 1369 & 17.378 & 14.282 & 16.42 & 14 & 13 \\
\hline 36 & LINALYL ACETATE & 0.5 & 1379 & 17.378 & 14.282 & 16.72 & 14 & 13 \\
\hline 37 & CisJasmone & 0.18 & 1393 & 17.378 & 14.282 & 17.15 & 14 & 13 \\
\hline 38 & trans-Caryophyllene & 0.27 & 1412 & 20.409 & 17.378 & 17.75 & 15 & 14 \\
\hline 39 & GERMACRENE-D & 0.27 & 1474 & 20.409 & 17.378 & 19.61 & 15 & 14 \\
\hline 40 & delta.-Cadinene & 0.18 & 1516 & 23.333 & 20.409 & 20.87 & 16 & 15 \\
\hline 41 & Caryophyllene oxide & 0.15 & 1574 & 23.333 & 20.409 & 22.57 & 16 & 15 \\
\hline 42 & alpha-thujene & 0.05 & 930 & 5.349 & 3.39 & 3.97 & 10 & 9 \\
\hline 43 & ALPHA-PINENE & 3.19 & 937 & 5.349 & 3.39 & 4.11 & 10 & 9 \\
\hline 44 & Verbenene & 0.03 & 956 & 5.349 & 3.39 & 4.488 & 10 & 9 \\
\hline 45 & Sabinene & 0.51 & 975 & 5.349 & 3.39 & 4.86 & 10 & 9 \\
\hline 46 & BETA-PINENE & 0.12 & 979 & 5.349 & 3.39 & 4.94 & 10 & 9 \\
\hline 47 & Heptanol & 0.08 & 988 & 5.349 & 3.39 & 5.117 & 10 & 9 \\
\hline 48 & BETA-MYRCENE & 0.18 & 992 & 5.349 & 3.39 & 5.197 & 10 & 9 \\
\hline 49 & gama-terpinene & 0.33 & 1006 & 7.991 & 5.349 & 5.518 & 11 & 10 \\
\hline 50 & alpha-terpinen & 0.07 & 1017 & 7.991 & 5.349 & 5.81 & 11 & 10 \\
\hline 51 & p-Cymene & 0.21 & 1025 & 7.991 & 5.349 & 6.004 & 11 & 10 \\
\hline \multirow[t]{2}{*}{52} & LINALOOL & 20.2 & 1032 & 7.991 & 5.349 & 6.187 & 11 & 10 \\
\hline & Total & 83 & & & & & & \\
\hline
\end{tabular}


Table3. Compositions of Artemisia kermanensis

\begin{tabular}{|c|c|c|c|c|c|c|c|c|}
\hline No & Compositions & $\%$ & RI & Sn+1 & Sn & RT & $C n+1$ & Cn \\
\hline 1 & artemisiatriene & 0.41 & 926 & 5.349 & 3.39 & 3.893 & 10 & 9 \\
\hline 2 & ALPHA-PINENE & 0.54 & 934 & 5.349 & 3.39 & 4.064 & 10 & 9 \\
\hline 3 & Camphene & 0.93 & 949 & 5.349 & 3.39 & 4.345 & 10 & 9 \\
\hline 4 & Verbenene & 1.88 & 954 & 5.349 & 3.39 & 4.448 & 10 & 9 \\
\hline 5 & Benzaldehyde & 0.11 & 960 & 5.349 & 3.39 & 4.562 & 10 & 9 \\
\hline 6 & BETA-PINENE & 0.08 & 977 & 5.349 & 3.39 & 4.9 & 10 & 9 \\
\hline 7 & p-menthatriene & 0.57 & 993 & 5.349 & 3.39 & 5.203 & 10 & 9 \\
\hline 8 & yomogi alcohol & 2.67 & 1001 & 7.991 & 5.349 & 5.375 & 11 & 10 \\
\hline 9 & alpha.-Terpinene & 0.2 & 1016 & 7.991 & 5.349 & 5.77 & 11 & 10 \\
\hline 10 & PARA CYMENE & 1.88 & 1024 & 7.991 & 5.349 & 5.97 & 11 & 10 \\
\hline 11 & 1,8-Cineole & 1.82 & 1030 & 7.991 & 5.349 & 6.142 & 11 & 10 \\
\hline 12 & Artemisia Ketone, & 0.11 & 1032 & 7.991 & 5.349 & 6.204 & 11 & 10 \\
\hline 13 & trans-Carane & 0.13 & 1050 & 7.991 & 5.349 & 6.674 & 11 & 10 \\
\hline 14 & gama-terpinene & 0.41 & 1056 & 7.991 & 5.349 & 6.828 & 11 & 10 \\
\hline 15 & Artemesia alcohol & 1.48 & 1082 & 7.991 & 5.349 & 7.526 & 11 & 10 \\
\hline 16 & Styrene, & 0.82 & 1087 & 7.991 & 5.349 & 7.658 & 11 & 10 \\
\hline 17 & alpha.-Thujone & 13.83 & 1108 & 11.075 & 7.991 & 8.253 & 12 & 11 \\
\hline 18 & Beta-Thujone & 6.23 & 1117 & 11.075 & 7.991 & 8.522 & 12 & 11 \\
\hline 19 & trans-Pinocarveol & 1.39 & 1138 & 11.075 & 7.991 & 9.163 & 12 & 11 \\
\hline 20 & Camphor & 4.13 & 1142 & 11,075 & 7.991 & 9.289 & 12 & 11 \\
\hline 21 & Camphore & 10.23 & 1144 & 11.075 & 7.991 & 9.363 & 12 & 11 \\
\hline 22 & p-Menth-1,5-dien-8-ol & 2.04 & 1147 & 11.075 & 7.991 & 9.455 & 12 & 11 \\
\hline 23 & 1-Menthene & 0.49 & 1156 & 11.075 & 7.991 & 9.712 & 12 & 11 \\
\hline 24 & Pinocarvone & 1.37 & 1160 & 11.075 & 7.991 & 9.838 & 12 & 11 \\
\hline 25 & Borneol & 1.97 & 1164 & 11.075 & 7.991 & 9.952 & 12 & 11 \\
\hline 26 & p-Mentha-1,5-dien-8-ol & 4.38 & 1166 & 11.075 & 7.991 & 10.021 & 12 & 11 \\
\hline 27 & Terpinene-4-ol & 1.01 & 1175 & 11.075 & 7.991 & 10.307 & 12 & 11 \\
\hline 28 & Naphthalene & 0.73 & 1178 & 11.075 & 7.991 & 10.393 & 12 & 11 \\
\hline 29 & p-Cymen-3-ol & 1.26 & 1182 & 11.075 & 7.991 & 10.519 & 12 & 11 \\
\hline 30 & alpha.-Terpineol & 0.72 & 1188 & 11.075 & 7.991 & 10.691 & 12 & 11 \\
\hline 31 & Verbenone & 1.53 & 1206 & 14.282 & 11.075 & 11.274 & 13 & 12 \\
\hline 32 & Norbornane & 0.36 & 1215 & 14.282 & 11.075 & 11.543 & 13 & 12 \\
\hline 33 & Cuminic aldehyde & 1.1 & 1235 & 14.282 & 11.075 & 12.19 & 13 & 12 \\
\hline 34 & (+)-Carvone & 0.48 & 1239 & 14.282 & 11.075 & 12.321 & 13 & 12 \\
\hline 35 & Carvotanacetone & 0.28 & 1243 & 14.282 & 11.075 & 12.441 & 13 & 12 \\
\hline 36 & CIS-MYRTANOL & 0.15 & 1247 & 14.282 & 11.075 & 12.579 & 13 & 12 \\
\hline 37 & Carvenone & 0.12 & 1253 & 14.282 & 11.075 & 12.762 & 13 & 12 \\
\hline 38 & Chrysanthenyl Acetate & 1 & 1256 & 14.282 & 11.075 & 12.882 & 13 & 12 \\
\hline 39 & Cinnamic aldehyde-E & 0.16 & 1264 & 14.282 & 11.075 & 13.134 & 13 & 12 \\
\hline 40 & Bornyl acetate & 2.3 & 1280 & 17.378 & 14.282 & 13.654 & 14 & 13 \\
\hline 41 & Thymol & 1.29 & 1286 & 17.378 & 14.282 & 13.86 & 14 & 13 \\
\hline 42 & Carvacrol & 1.78 & 1297 & 17.378 & 14.282 & 14.175 & 14 & 13 \\
\hline 43 & alpha.-Copaene & 0.23 & 1368 & 20.409 & 17.378 & 16.412 & 15 & 14 \\
\hline 44 & Methyl cinnamate & 0.15 & 1375.7 & 20.409 & 17.378 & 16.641 & 15 & 14 \\
\hline 45 & (Z)-Jasmone & 0.22 & 1393.1 & 20.409 & 17.378 & 17.168 & 15 & 14 \\
\hline 46 & Methyleugenol & 0.15 & 1399.3 & 20.409 & 17.378 & 17.357 & 15 & 14 \\
\hline 47 & trans-Caryophyllene & 0.3 & 1395.6 & 23.333 & 20.409 & 17.746 & 16 & 15 \\
\hline 48 & Alpha.-Curcumen & 0.15 & 1475.4 & 23.333 & 20.409 & 19.691 & 16 & 15 \\
\hline 49 & Spathulenol & 0.25 & 1569 & 23.333 & 20.409 & 22.426 & 16 & 15 \\
\hline 50 & Caryophyllene & 0.07 & 1644.5 & 26.141 & 23.333 & 24.583 & 17 & 16 \\
\hline
\end{tabular}


Table 4. Compositions of Lavandula officinalis

\begin{tabular}{|c|c|c|c|c|c|c|c|c|}
\hline $\begin{array}{l}\mathbf{N} \\
\mathbf{0}\end{array}$ & Compositions & $\%$ & $\mathbf{R} \mathbf{I}$ & $\mathrm{Sn}+1$ & Sn & RT & $C n+1$ & Cn \\
\hline 1 & ALPHA-PINENE & 7.58 & 938 & 5.349 & 3.39 & 4.139 & 10 & 9 \\
\hline 2 & CAMPHENE & 4.51 & 952 & 5.349 & 3.39 & 4.408 & 10 & 9 \\
\hline 3 & Verbenene & 0.64 & 956 & 5.349 & 3.39 & 4.488 & 10 & 9 \\
\hline 4 & 1,3,5-Cycloheptatriene & 0.03 & 972 & 5.349 & 3.39 & 4.808 & 10 & 9 \\
\hline 5 & BETA-PINENE & 0.49 & 979 & 5.349 & 3.39 & 4.94 & 10 & 9 \\
\hline 6 & 3-OCTANONE & 2.19 & 988 & 5.349 & 3.39 & 5.123 & 10 & 9 \\
\hline 7 & BETA-MYRCENE & 1.18 & 993 & 5.349 & 3.39 & 5.209 & 10 & 9 \\
\hline 8 & 3 OCTANOL & 0.36 & 997 & 5.349 & 3.39 & 5.295 & 10 & 9 \\
\hline 9 & AlphaPhellandrene & 0.05 & 1007 & 7.991 & 5.349 & 5.523 & 11 & 10 \\
\hline 10 & o-Isopropenyltoluene & 0.08 & 1014 & 7.991 & 5.349 & 5.712 & 11 & 10 \\
\hline 11 & AlphaTerpinene & 0.12 & 1017 & 7.991 & 5.349 & 5.81 & 11 & 10 \\
\hline 12 & p-Cymene & 2.96 & 1025 & 7.991 & 5.349 & 6.01 & 11 & 10 \\
\hline 13 & 1,8-Cineol & 12.01 & 1033 & 7.991 & 5.349 & 6.222 & 11 & 10 \\
\hline 14 & gamma.-Terpinene & 0.08 & 1057 & 7.991 & 5.349 & 6.851 & 11 & 10 \\
\hline 15 & Linalool Oxide & 0.06 & 1072 & 7.991 & 5.349 & 7.24 & 11 & 10 \\
\hline 16 & Methyl banzoate & 0.99 & 1088 & 7.991 & 5.349 & 7.669 & 11 & 10 \\
\hline 17 & Linalool & 2.45 & 1100 & 11.075 & 7.991 & 8.001 & 12 & 11 \\
\hline 18 & Thujancis & 0.81 & 1103 & 11.075 & 7.991 & 8.098 & 12 & 11 \\
\hline 19 & D-Fenchyl alcohol & 0.28 & 1112 & 11.075 & 7.991 & 8.373 & 12 & 11 \\
\hline 20 & Pinocarveol & 0.12 & 1138 & 11.075 & 7.991 & 9.157 & 12 & 11 \\
\hline 21 & Camphore & 9.16 & 1144 & 11.075 & 7.991 & 9.346 & 12 & 11 \\
\hline 22 & Isopinocamphone & 1.72 & 1158 & 11.075 & 7.991 & 9.775 & 12 & 11 \\
\hline 23 & Pinocarvone & 0.13 & 1160 & 11.075 & 7.991 & 9.832 & 12 & 11 \\
\hline 24 & Pinocamphone & 0.39 & 1171 & 11.075 & 7.991 & 10.193 & 12 & 11 \\
\hline 25 & Terpene-4-ol & 1.27 & 1174 & 11.075 & 7.991 & 10.284 & 12 & 11 \\
\hline 26 & naphtalene & 0.08 & 1177 & 11.075 & 7.991 & 10.378 & 12 & 11 \\
\hline 27 & p-Cymen-8-ol & 0.23 & 1183 & 11.075 & 7.991 & 10.553 & 12 & 11 \\
\hline 28 & AlphaTerpineol & 2.31 & 1188 & 11.075 & 7.991 & 10.702 & 12 & 11 \\
\hline 29 & Myrtenol & 0.35 & 1194 & 11.075 & 7.991 & 10.896 & 12 & 11 \\
\hline 30 & no pol (terpene) & 1.11 & 1203 & 14.282 & 11.075 & 11.182 & 13 & 12 \\
\hline 31 & Verbenone & 8.47 & 1209 & 14.282 & 11.075 & 11.366 & 13 & 12 \\
\hline 32 & trans-Carveol & 0.13 & 1215 & 14.282 & 11.075 & 11.554 & 13 & 12 \\
\hline 33 & beta.-Citronellol & 0.1 & 1225 & 14.282 & 11.075 & 11.869 & 13 & 12 \\
\hline 34 & Pulegone & 0.09 & 1235 & 14.282 & 11.075 & 12.195 & 13 & 12 \\
\hline 35 & Piperitone & 0.04 & 1250 & 14.282 & 11.075 & 12.67 & 13 & 12 \\
\hline 36 & Cinnamaldehyde & 0.05 & 1265 & 14.282 & 11.075 & 13.145 & 13 & 12 \\
\hline 37 & Borneol acetate & 2.41 & 1281 & 14.282 & 11.075 & 13.66 & 13 & 12 \\
\hline 38 & Thymol & 6.23 & 1288 & 14.282 & 11.075 & 13.906 & 13 & 12 \\
\hline 39 & $\begin{array}{c}\text { 2-Hydroxy-4-Isopropyl-1- } \\
\text { Methylbenzene }\end{array}$ & 0.17 & 1290 & 14.282 & 11.075 & 13.975 & 13 & 12 \\
\hline 40 & Carvacrol & 4.14 & 1297 & 14.282 & 11.075 & 14.192 & 13 & 12 \\
\hline 41 & alpha.-Terpinene & 0.15 & 1329 & 17.378 & 14.282 & 15.194 & 14 & 13 \\
\hline 42 & PIPERITENONE & 0.37 & 1335 & 17.378 & 14.282 & 15.36 & 14 & 13 \\
\hline 43 & alpha.-Cubebene & 0.06 & 1343 & 17.378 & 14.282 & 15.617 & 14 & 13 \\
\hline 44 & Thymyl acetate & 0.06 & 1349 & 17.378 & 14.282 & 15.789 & 14 & 13 \\
\hline 45 & alpha.-Copaene & 0.43 & 1369 & 17.378 & 14.282 & 16.424 & 14 & 13 \\
\hline 46 & trans-Caryophyllene & 0.47 & 1412 & 20.409 & 17.378 & 17.751 & 15 & 14 \\
\hline 47 & alpha.-Humulene & 0.22 & 1446 & 20.409 & 17.378 & 18.781 & 15 & 14 \\
\hline 48 & Farnesene & 0.08 & 1451 & 20.409 & 17.378 & 18.93 & 15 & 14 \\
\hline 49 & eta.-Acoradiene & 0.09 & 1460 & 20.409 & 17.378 & 19.182 & 15 & 14 \\
\hline 50 & gamma.-Cadinene & 0.16 & 1473 & 20.409 & 17.378 & 19.599 & 15 & 14 \\
\hline 51 & Zingiberene & 0.1 & 1488 & 20.409 & 17.378 & 20.034 & 15 & 14 \\
\hline
\end{tabular}




\begin{tabular}{|c|c|c|c|c|c|c|c|c|}
\hline 52 & .beta.-Himachalene & 0.28 & 1492 & 20.409 & 17.378 & 20.166 & 15 & 14 \\
\hline 53 & delta.-Cadinene & 0.27 & 1516 & 23.333 & 20.409 & 20.881 & 16 & 15 \\
\hline 54 & Alpha-Cedrene & 0.15 & 1524 & 23.333 & 20.409 & 21.116 & 16 & 15 \\
\hline 55 & Germacrene B & 0.06 & 1548 & 23.333 & 20.409 & 21.814 & 16 & 15 \\
\hline 56 & spathulenol & 0.26 & 1567 & 23.333 & 20.409 & 22.375 & 16 & 15 \\
\hline 57 & Caryophyllene oxide & 0.29 & 1572 & 23.333 & 20.409 & 22.518 & 16 & 15 \\
\hline 58 & AlphaFarnesene & 0.06 & 1587 & 23.333 & 20.409 & 22.941 & 16 & 15 \\
\hline 59 & ButlidenePhthalide & 0.15 & 1642 & 26.141 & 23.333 & 24.515 & 17 & 16 \\
\hline 60 & 3N ButylPhthalide & 4.62 & 1687 & 26.141 & 23.333 & 25.773 & 17 & 16 \\
\hline 61 & ButylideneDihydro-Phthalide & 0.09 & 1720 & 28.733 & 26.141 & 26.66 & 18 & 17 \\
\hline 62 & ALPHA-PINENE & 7.58 & 938 & 5.349 & 3.39 & 4.139 & 10 & 9 \\
\hline 63 & CAMPHENE & 4.51 & 952 & 5.349 & 3.39 & 4.408 & 10 & 9 \\
\hline 64 & Verbenene & 0.64 & 956 & 5.349 & 3.39 & 4.488 & 10 & 9 \\
\hline 65 & 1,3,5-Cycloheptatriene & 0.03 & 972 & 5.349 & 3.39 & 4.808 & 10 & 9 \\
\hline 66 & BETA-PINENE & 0.49 & 979 & 5.349 & 3.39 & 4.94 & 10 & 9 \\
\hline 67 & 3-OCTANONE & 2.19 & 988 & 5.349 & 3.39 & 5.123 & 10 & 9 \\
\hline 68 & BETA-MYRCENE & 1.18 & 993 & 5.349 & 3.39 & 5.209 & 10 & 9 \\
\hline \multirow[t]{2}{*}{69} & 3 OCTANOL & 0.36 & 997 & 5.349 & 3.39 & 5.295 & 10 & 9 \\
\hline & Total & & & & & & & \\
\hline
\end{tabular}

Table 5. Compositions of Eucalyptus caesia

\begin{tabular}{|c|c|c|c|c|c|c|c|c|}
\hline No & Compositions & $\%$ & $\mathbf{R I}$ & $S n+1$ & Sn & RT & $C n+1$ & Cn \\
\hline 1 & ALPHA-Thujan & 0.3 & 929 & 5.349 & 3.39 & 3.967 & 10 & 9 \\
\hline 2 & ALPHA-PINENE & 7.7 & 937 & 5.349 & 3.39 & 4.116 & 10 & 9 \\
\hline 3 & CAMPHENE & 0.09 & 951 & 5.349 & 3.39 & 4.385 & 10 & 9 \\
\hline 4 & Sabinene & 0.08 & 975 & 5.349 & 3.39 & 4.86 & 10 & 9 \\
\hline 5 & BETA-PINENE & 0.7 & 979 & 5.349 & 3.39 & 4.94 & 10 & 9 \\
\hline 6 & BETA-MYRCENE & 0.63 & 992 & 5.349 & 3.39 & 5.197 & 10 & 9 \\
\hline 7 & ALPHA. TERPINENE & 0.2 & 1018 & 7.991 & 5.349 & 5.821 & 11 & 10 \\
\hline 8 & $\mathrm{p}$-Cymene & 14.11 & 1029 & 7.991 & 5.349 & 6.107 & 11 & 10 \\
\hline 9 & 1,8-CINEOL & 40.18 & 1034 & 7.991 & 5.349 & 6.244 & 11 & 10 \\
\hline 10 & gamma.-Terpinene & 12.43 & 1059 & 7.991 & 5.349 & 6.92 & 11 & 10 \\
\hline 11 & ALPHA. TERPINENOL & 1.74 & 1087 & 7.991 & 5.349 & 7.652 & 11 & 10 \\
\hline 12 & LINALOOL & 0.13 & 1099 & 11.075 & 7.991 & 7.967 & 12 & 11 \\
\hline 13 & FENCHYL ALCOHOL & 0.07 & 1112 & 11.075 & 7.991 & 8.361 & 12 & 11 \\
\hline 14 & trans-Pinocarveol & 0.62 & 1136 & 11.075 & 7.991 & 9.088 & 12 & 11 \\
\hline 15 & MENTHOFURAN & 0.16 & 1159 & 11.075 & 7.991 & 9.815 & 12 & 11 \\
\hline 16 & BORNEOL & 0.13 & 1162 & 11.075 & 7.991 & 9.901 & 12 & 11 \\
\hline 17 & Terpinene-4-ol & 5.62 & 1174 & 11.075 & 7.991 & 10.284 & 12 & 11 \\
\hline 18 & p-Cymen-8-ol & 0.72 & 1181 & 11.075 & 7.991 & 10.502 & 12 & 11 \\
\hline 19 & MENTHOL & 1.07 & 1184 & 11.075 & 7.991 & 10.576 & 12 & 11 \\
\hline 20 & ALPHA. TERPINEOL & 1.53 & 1187 & 11.075 & 7.991 & 10.679 & 12 & 11 \\
\hline 21 & trans-Carveol & 0.77 & 1214 & 14.282 & 11.075 & 11.526 & 13 & 12 \\
\hline 22 & cis-Carveol & 0.41 & 1226 & 14.282 & 11.075 & 11.898 & 13 & 12 \\
\hline 23 & Carvone & 0.25 & 1239 & 14.282 & 11.075 & 12.31 & 13 & 12 \\
\hline 24 & GERANIOL & 0.52 & 1249 & 14.282 & 11.075 & 12.653 & 13 & 12 \\
\hline 25 & Thymol & 0.51 & 1280 & 14.282 & 11.075 & 13.637 & 13 & 12 \\
\hline 26 & Carvacrol ETHYL ETHER & 0.52 & 1288 & 14.282 & 11.075 & 13.912 & 13 & 12 \\
\hline 27 & Carvacrol & 0.41 & 1295 & 14.282 & 11.075 & 14.112 & 13 & 12 \\
\hline
\end{tabular}


Table 6. Compositions of Rosemarinus officinalis

\begin{tabular}{ccccccccc}
\hline No & Compositions & \% & RI & Sn+1 & Sn & RT & Cn+1 & Cn \\
\hline 1 & alpha-pinene & 23.93 & 942.318 & 5.349 & 3.39 & 4.219 & 10 & 9 \\
2 & Camphen & 8.7 & 955.436 & 5.349 & 3.39 & 4.476 & 10 & 9 \\
3 & Vernenen & 1.3 & 959.826 & 5.349 & 3.39 & 4.562 & 10 & 9 \\
4 & 3-Octanone & 5.63 & 991.679 & 5.349 & 3.39 & 5.186 & 10 & 9 \\
5 & P-cymene & 7.48 & 1026.95 & 7.991 & 5.349 & 6.061 & 11 & 10 \\
6 & Limonene & 2.99 & 1031.3 & 7.991 & 5.349 & 6.176 & 11 & 10 \\
7 & P-cymenene & 1.13 & 1089.33 & 7.991 & 5.349 & 7.709 & 11 & 10 \\
8 & Camphor & 10.97 & 1144.39 & 11.075 & 7.991 & 9.36 & 12 & 11 \\
9 & Naphtalene & 0.32 & 1178.44 & 11.075 & 7.991 & 10.41 & 12 & 11 \\
10 & p-Cymen-8-ol & 0.36 & 1182.72 & 11.075 & 7.991 & 10.542 & 12 & 11 \\
11 & Verbenon & 15.44 & 1208.57 & 14.282 & 11.075 & 11.35 & 13 & 12 \\
\hline \multicolumn{2}{c}{ Total 78.25 } \\
\hline
\end{tabular}

Comparing various essential oils revealed that at $24 \mathrm{~h}$ post incubation, the lowest dose of essential oils showed the same inhibition zone ( $p>0.10$ ), but increasing essential oil to $50000 \mathrm{ppm}$ has resulted in highest antimicrobial activity for thymus vulgaris followed by Rosemarinus Officinalis and Lavandula Officinalis $(13,71,9,42$ and $6.16 \mathrm{~mm})$ respectively. As it is shown in Figure 2, at highest concentration of essential oils (400000), Thymus vulgaris was as effective as two positive controls gentamicin and Chloramphenicol in inhibiting microbial growth. Other essential oils had lower antimicrobial activity and at 48 and $72 \mathrm{~h}$ post incubation, the same response was observed.

Historically, many plant oils and extracts have been used as topical antiseptics and reported to have antimicrobial properties. This specific characteristics, beside of other health benefit properties of herbs, make them suitable candidate for use in the pharmacological usage. In recent years, many researchers investigated antimicrobial activity of many herbal plants, or their bioactive compounds as possible alternatives to chemically synthetic antimicrobial drugs to which many pathogenic microorganisms have become resistant. Various researches have documented the antimicrobial activity of essential oils and plant extracts including Artemisia kermanensis Podl, Eucalyptus caesia, thymus vulgaris, Lavandula officinalisand Rosmarinus officinalis (Bayoub et al., 2010, Figueiredo et al., 2008, Fu et al., 2007, Ghalem and Mohamed, 2008, Stojanović-Radić et al., 2010). The medicinal plants have been long used against the growth of bacteria and several studies have been conducted on the effects of these plants (Gavanji et al., 2012a). The results of $T$. vulgaris analysis showed that more than 42 chemical compounds have been identified (Table 2) that $87.83 \%$ of which constitute the essential components. The major components of the essential oil are made by Carvacrol $(43.42 \%)$ and other major compounds are ALPHA-PINENE (3.19\%), LINALOOL (20,22\%), Carvone (1.45\%) and Isomenthone (4.92\%). Carvacrol and Thymol are two phenolic compounds found in T. vulgaris which their strong antimicrobial effect has been revealed by researchers in several studies (Vernozy-Rozand et al., 2002, Özkan et al., 2003).

Studies in 2003 showed that the T. vulgaris essential oil using Disc Diffusion method at the concentration of $0.4 \%$ has antimicrobial activity on E.coli. Also Burt and his coworker in 2003 showed that the $T$. vulgaris essential oil in low concentrations $(0.12 \%$ and $0.25 \%)$ possess Bacteriostatic and bactericidal effects respectively (Burt and Reinders, 2003). As the oil possess higher degree of phenolic material, it has stronger antimicrobial properties. These materials include Carvacrol, Eugenol and Thymol (Burt, 2004). Also it has been proven that essential components interact with each other and play an important role in determining the antimicrobial effect of the plant. Carvacrol and Thymol have Synergistic effects (Didry et al., 1994). R. officinalis is another plant studied in this experiment. The chemical analysis of rosemary essential oil by GC has identified 11 compounds (Table 6) which they make $78.25 \%$ rosemary essential oil. Major components of the essential oil include alpha-pinene (23.93\%), Camphen (8.7\%), Camphor (10.97\%), Verbenon (15.44\%), P-cymene (7.48\%) and 3-Octanone (5.63\%). Usually essential oils which are rich in phenolic compounds show significant antimicrobial properties. In fact the phenolic compounds presented in the essential oils create the most effective 
antimicrobial properties. These compounds can penetrate into the cell membrane and the cell contents could have been involved in clotting (Gavanji et al., 2012b, Minnunni et al., 1992). In general, terpenes may have other different antimicrobial mechanisms. The investigations have also revealed that the essential oils may disrupt energy production and synthesis of structural components of the yeast enzyme activity system (Conner and Beuchat, 1984). Results obtained from A.kermanensis showed more than 50 chemical compounds (Table 3 ) in which $75.84 \%$ is components of essential oil. The major compounds of the essential oil are p-Menth-1, 5-dien-8-ol (4.38\%), Camphore (14.36\%) and BetaThujone (6.23\%). The essential oil of various species of Artemisia kermanensis have effective antibacterial activities against bacteria such as oureus, Staphylococcus, E. coli and Staphylococcus epidermidis as well as against yeast Crypto coccus, Candida albicans and dermatophytes fungi such as Canis, Microsporum, Microsporum gypseum, Fonsecaea pedrosoi andTrichophyton rubrum (Lopez- Lutz et al., 2008). According to some studies, the amount of 1,8-Cineole in specie of Eucalyptusglobuls in Uruguay country was reported $64.5 \%$ (Dellacasa et al., 1990). Also this component in Cuba country was reported between 75 to $77 \%$ (Magraner Hernandez et al., 1988). Also in California it is equal to $86.67 \%$ (Nishimura and Calvin, 1979), in Morocco is between 58 to 82\% (Zrira and Benjilali, 1996) and in South Africa it is reported $48.7 \%$ (Thilivahalt et al., 1986). The overall quality and quantity of the essential oil of particular species vary according to season, geographical location and the location of plants. In some species, the essence is well made in warm and sunny season. Climate and soil conditions can affect the composition of the oil (Arnold et al., 1997). Based on results obtained from E. caesia Benth in Khuzestan province (Iran), the analysis of 1,8 -CINEOL by GC system was reported $69.4 \%$.

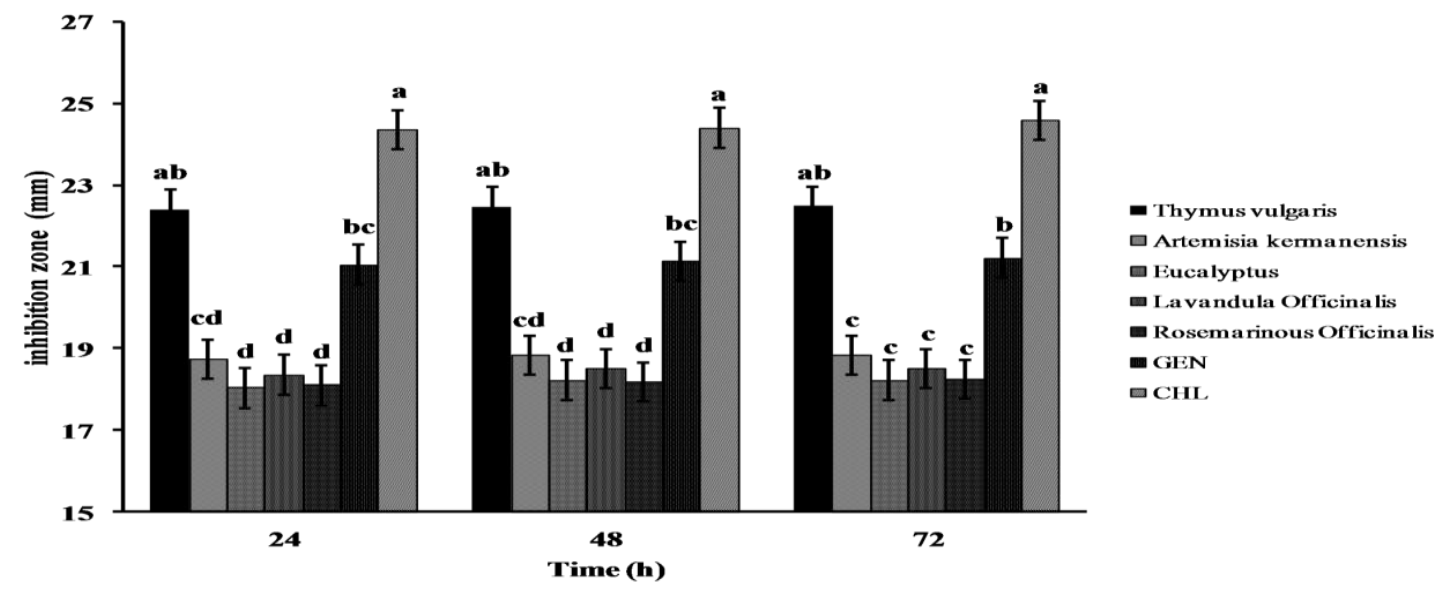

Figure 2. The highest concentration of essential oils of treatment $(400000 \mathrm{ppm})$ in comparison to two positive control antimicrobial agent gentamicin (GEN) and Chloramphenicol (CHL) in inhibiting microbial growth. Same letters in each column show no significantly different existed at $p<0.05$.

\section{References}

Adams R.P. (2005), Identification of Essential oil Components by Gas Chromatography-quadropole Mass spectroscopy, J. Am. Soc. Mass Spectrom, 16, 1902-1903.

Al-Bayati F.A. (2008), Synergistic antibacterial activity between Thymus vulgaris and Pimpinella anisum essential oils and methanol extracts, J Ethnopharmacol., 116, 403-406.

Arnold V., Valentine G. and Bellomaria B. (1997), Comparative study of the essential oils from Rosmarinus eiocalyx \& four algeria and R. officinalis L. from other countries, Essent Oil Res, 9, 167-175.

Bakkali F., Averbeck S., Averbeck D. and Idaomar M. (2008), Biological effects of essential oils - A review, Food Chem. Toxicol., 46,446-475. 
Bayoub K., Baibai T.,Mountassif D.,Retmane A. and Soukri A. (2010), Antibacterial activities of the crude ethanol extracts of medicinal plants against Listeria monocytogenes and some other pathogenic strains, Afr. J. Biotechnol., 9, 4251-4258.

Burt S. (2004), Essential oils: their antibacterial properties and potential applications in foods a review, Int. J. Food Microbiol., 94, 223-253.

Burt S.A. and Reinders R.D. (2003), Antibacterial activity of selected plant essential oils against Escherichia coli 0157:H7, Lett. Appl. Microbiol., 36, 162-167.

Conner D.E. and Beuchat L.R. (1984), Effects of essential oils from plants on growth of food spoilage yeasts, J. Food Sci. , 49, 429-434.

Delaquis P.J., Stanich K., Girard B. and Mazza G. (2002), Antimicrobial activity of individual and mixed fractions of dill, cilantro, coriander and eucalyptus essential oils, Int. J. Food Microbiol.,74, 101-109.

Dellacasa E., Menendez P., Moyna P. and Soler E. (1990), Chemical composition of Eucalyptus essential oils grown in Uruguay, Flavour and Fragrance J, 5,91-98.

Di Pasqua R., Betts G., Hoskins N., Edwards M., Ercolini D. and Mauriello G. (2007), Membrane toxicity of antimicrobial compounds from essential oils, J. Agric. Food Chem., 55, 4863-4870.

Didry N., Dubreuil L. and Pinkas M. (1994), Activity of thymol, carvacrol, cinnamaldehyde and eugenole on oral bacterial, Pharm Acta Helv., 69, 25-28.

Figueiredo A.C., Barroso J.G., Pedro L.G. and Scheffer J.C. (2008), Factors affecting secondary metabolite production in plants: volatile components and essential oils, Flavour Frag J, 23, 213-226.

Fu Y.,Zu Y., Chen L., Shi X., Wang Z., Sun S. and Efferth T. (2007), Antimicrobial Activity of Clove and Rosemary Essential Oils Alone and in Combination, Phytother. Res, 21, 989-994.

Gavanji S., Asgari M.J., Vaezi R. and Larki B. (2011), Antifungal effect of the extract of propolis on the growth of three species of Epidermophyton $f$ lucosum, Trichophyton violaseum and Trichophytontonsorans in laboratory environment, Afr J Pharm Pharmacol, 5, 2642-2646.

Gavanji S., Larki B., Doostmohammadi M. and Mortezaeinezad F. (2012a), Production of a new mixed herbal medicine for minor Aphthous Ulcers, Medicinal Plants, 4, 49-51.

Gavanji S., Larki B., Jalali Zand A., Mohammadi E., Mehrasa M. and Taraghian A.M. (2012b), Comparative effects of propolis of honey bee on pathogenic bacteria, Afr J Pharm Pharmacol, 6, 2408-2412.

Ghalem B.R. and Mohamed B. (2008), Antibacterial activity of leaf essential oils of Eucalyptus globulus and Eucalyptus camaldulensis, Afr J Pharm Pharmacol, 2, 211-215.

Kazemi M., Dakhili M.,Dadkhah A.,Yasrebifar Z. and Larijani K. (2011), Composition, antimicrobial and antioxidant activities of the essential oil of Artemisia kermanensis Podl., an endemic species from Iran, J Med Plants Res, 5, 4481-4486.

Lopez- Lutz D., Alviano S.D., Alviano S.C. and Kolodziejczyk P.P. (2008), Screening of chemical composition of microbial and antioxidant activities of Artemisia essential oils, Flavour Frag J, 8, 131-137.

Magraner Hernandez J., Loret de mole L.M., Gra Rios G., Rasimbazafy M.and Rosado Perez A. (1988), Estudio de la composicion quimica del aceite essencial de eucalyptus globulus Labill ssp bicostata obtenido en Cuba, Rev Cub Farm, 22, 76- 85.

Minnunni M., Wolleb U., Mueller O., Pfeifer A. and Aeschbacher H.U. (1992), Natural antioxidants as inhibitors of oxygen species induced mutagenicity, Mutat Res., 269, 193-200.

Nishimura H. and Calvin M. (1979), Essential oil of Eucalyptus globulus in California, J Agri Food Chem, 27, $432-435$.

Orhan I.E., ÖZÇELIK B.,Kartal M. and Kan Y. (2012), Antimicrobial and antiviral effects of essential oils from selected Umbelliferae and Labiatae plants and individual essential oil components, Turk J Biol, 36, 239-246.

Özkan G., Sağdiç O. and Özcan M. (2003), Note: Inhibition of pathogenic bacteria by essential oils at different concentrations, Food Sci Technol Int, 9, 85-88.

Sahin F., Güllüce M., Daferera D., Sökmen A., Sökmen M., Polissiou M., Agar G. And Özer H. (2004), Biological activities of the essential oils and methanol extract of Origanum vulgare ssp. vulgare in the Eastern Anatolia region of Turkey, Food Control, 15, 549-557. 
Sparkman O.D. (1997), Identification of essential oil components by gas chromatography/quadrupole mass spectroscopy, J Am Soc Mass Spectrom, 8, 671-672.

Stojanović-Radić Z., Nešić M., Čomić L. and Radulović N. (2010), Antimicrobial activity and cytotoxicity of commercial rosemary essential oil (Rosmarinus officinalis L.), Biologica Nyssana, 1, 83-88.

Takahashi T., Kokubo R. and Sakaino M. (2004), Antimicrobial activities of eucalyptus leaf extracts and flavonoids from Eucalyptus maculata, Lett Appl Microbiol, 39, 60-64.

Telci I., Toncer O.G. and Sahbaz N. (2006), Yield, Essential Oil Content and Composition of Coriandrum sativum Varieties (var. vulgare Alef and var. microcarpum DC.) Grown in Two Different Locations, J Essent Oil Res,18, 189-193.

Thilivahalt N.T. and Von Wandruszka M.A. (1986), Essential oils of South African Eucalyptus species (Myrtaceae), $S$ Afr J Chem, 39, 95-100.

Van Den Dool H. and Kratz P.D. (1963), A generalization of the retention index system including linear temperature programmed gas-liquid partition chromatography, J. Chromatog, 11, 463-471

Vernozy-Rozand C., Ray-Gueniot S., Ragot C., Bavai C., Mazuy C., Montet M.P., Bouvet J.. and Richard Y. (2002), Prevalence of Escherichia coli 0157:H7 in industrial minced beef, Lett Appl Microbiol, 35, 7-11.

Zrira S.S. and Benjilali B.B. (1996), Seasonal changes in the volatile oil and cineole contents of five Eucalyptus species growing in the Morocco, J Essent Oil Res, 8, 19-24. 